\title{
Model Predictive Speed and Headway Control with Energy Optimization for a Series Hybrid Vehicle with Range Extender
}

\author{
Liting $\mathrm{Lu}^{1, \mathrm{a}}$ and Steffen Müller ${ }^{1}$ \\ ${ }^{1}$ Department of Automotive Engineering, Technische Universität Berlin, Berlin, Germany
}

\begin{abstract}
In this paper, a model predictive speed and headway control (MPSHC) with multi-input and multi-output (MIMO) is developed for a series hybrid vehicle with range extender (BEVx). The MPSHC calculates the optimal solutions of the efficient driving strategy and the efficient power supply, with the vehicle longitudinal dynamics and battery dynamics. It takes the driver's demand, the legal speed limit, the driving behavior of the preceding object vehicle, the topography and the state of charge $(\mathrm{SoC})$ into account. The energy consumption as the objective is minimized in the predictive horizon. The simulation results show that the proposed MPSHC algorithm has a good energy saving potential, compared with the conventional Adaptive Cruise Control (ACC) and the simple energy supply strategy.
\end{abstract}

\section{Introduction}

Nowadays the ACC driver assistance system keeps the set speed in speed control and copy the speed of the preceding object vehicle in order to keep the set distance in case of headway control. It reduces the driver's load and the risk of rear-end collision, but leads to a reduced electric range and unnecessary high fuel consumption, namely $\mathrm{CO}_{2}$ emissions, for series hybrid vehicles with range extender.

With the increase of the real-time information about the traffic and road as well as the advancement of microcomputers, there has been an increasing effort put on MPC based algorithms for vehicle control. In the MPSHC area for the conventional and pure electric vehicles, the energy consumption is optimized through the efficient behavior of driving. For example, in [1] and [2] the MPC is used for the speed and headway control for passenger cars with engine. They propose a MPC algorithm to find the fuel efficient speed trajectories with different road and traffic scenarios. A driver assistance system of predictive speed control with energy optimization for pure electric vehicles is presented in [3]. In the case of the hybrid vehicle the efficient charge/discharge strategy of the battery is additionally considered as shown in [4]. This contribution presents the MPSHC for a parallel hybrid vehicle with a small capacity battery. However it considers no prediction of the topography. In response to the mentioned contributions, this paper presents the MPSHC extended from the already proposed MPC structure shown in [1] and [2] for a series hybrid vehicle with range extender, which has a battery with a big capacity. In this paper the driver demand, the legal speed limit, the driving behavior of the preceding vehicle, the topography and the SoC are all considered. The MIMO optimal problem is formulated as the quadratic programming, and a numerical solver "MOSEK" for large-scale problem is utilized with good real-time capability.

\section{The Structure of the Powertrain of the Series Hybrid Vehicle with Range Extender}

Fig. 1 shows the reduced structure of the powertrain for the series hybrid vehicle with range extender.

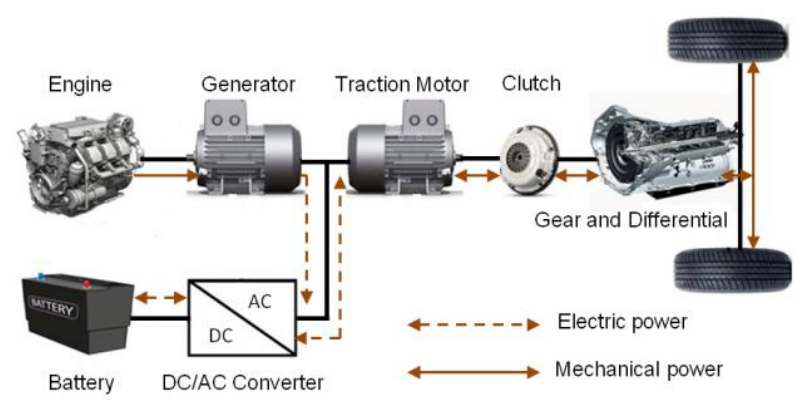

Figure 1. Powertrain structure and power flows for the BEVx

The combination of the downsized engine and the generator is called range extender module. The generated electrical energy by the range extender can either drive the electric traction motor or be saved in the lithium battery. The range extender module works along the optimal operation line (OOL), because there is no mechanical connection between the range extender module and the

\footnotetext{
${ }^{\mathrm{a}}$ Corresponding author: liting.lu@campus.tu-berlin.de
} 
driving wheel. The OOL on the brake specific fuel consumption (BSFC) map includes the optimal operation points with the best efficiencies for different output powers of the range extender module. Furthermore when the vehicle brakes with the regenerative brake, the kinetic energy is saved again in the battery.

\section{The Idea of the MPSHC}

\subsection{The Vehicle Model for the MPSHC}

The nonlinear equation of the longitudinal vehicle dynamics based on the energy theorem for the speed and headway control is written as follows,

$$
\begin{aligned}
& \frac{1}{2} m v(k+1)^{2}=\frac{1}{2} m v(k)^{2}-C_{w} \Delta s v(k)^{2}+ \\
& \frac{i}{r} T(k) \Delta s-\operatorname{mg} \Delta s \theta-\mu \mathrm{mg} \Delta \mathrm{s} .
\end{aligned}
$$

On the other hand, the related battery dynamics is expressed as below,

$$
\operatorname{soc}(k+1)=\operatorname{soc}(k)-\frac{p_{\mathrm{b}}(k)}{Q U_{0}} \frac{\Delta s}{v(k)} .
$$

The vehicle and battery parameters in Eq. 1 and Eq. 2 are the vehicle mass $m$, the acceleration of gravity $g$, the air drag coefficient $C_{w}$, the transmission ratio of the gear and differential $i$, the tire radius $r$, the rolling coefficient $\mu$, the total battery capacity $Q$ and the battery terminal voltage $U_{0}$. The dynamic parameters are the vehicle speed $v$, the torque of the traction motor $T$, the road grade $\theta$, the SoC and the battery power $p_{b}$. The above equations are discretized with respect to the road segment $\Delta s$.

However in order to adopt the real-time capable numerical solver for the MPC online calculation, the system model consisting of Eq. 1 and Eq. 2 is formulated as linear model written below,

$$
\left\{\begin{array}{c}
x_{1}(k+1)=a x_{1}(k)+b_{T} u_{1}(k)+b_{\theta} \theta(k)-\gamma \\
x_{2}(k+1)=x_{2}(k)+\frac{c}{v_{\mathrm{p}}} u_{2}(k)
\end{array}\right.
$$

In Eq. 3 the first state $x_{1}$ is the speed squared $v^{2}$ and the first control input $u_{1}$ is the torque of the traction motor $T$. The road grade $\theta$ is the disturbance and $\gamma$ is the rolling resistance. The state $x_{2}$ is the $\mathrm{SoC}$ and the second control input $u_{2}$ is the battery power $p_{b}$. The parameter $v_{\mathrm{p}}$ is the speed reference which is demonstrated in Eq. 14 in the subsection "Optimization Constraints", in which the original speed $v$ in Eq. 2 is replaced, in order to formulate the equation linearly.

\subsection{Optimal Cost Function.}

Because the battery in the BEVx has a big capacity, the processes of charge-depleting (CD) and charge-sustaining (CS) must not always simultaneously be included in a relative short predictive horizon of MPSHC. In this paper they are discussed separately. In the CD mode the level of SoC is higher than the lower limit and the battery supplies the electrical energy to the traction motor alone. In contrast, in the CS mode, the lower limit is reached and the $\mathrm{SoC}$ is maintained around the lower limit by the range extender or the regenerative brake.

For the CD mode the optimal cost function has the following form,

$$
J=\sum_{k=1}^{n}\left(e(k)^{2}+\rho\left(u_{1}(k)-u_{\mathrm{L}}(k)\right)^{2}\right) .
$$

In Eq. 4 the variable $e$ denotes electrical energy consumption by the traction motor within $\Delta s$. In order to minimize uncomfortable abrupt acceleration, another term $\rho\left(u_{1}(k)-u_{L}(k)\right)$ related to the deviations between the consequent driving torques, is included in the cost function with the weight $\rho$ [1]. Here, as well as later in the cost function of CS mode, the squared terms are used to avoid the chattering in the optimal solution by the linear programming.

The electrical energy consumption $e$ in the optimal cost function is explained as follows in detail,

$$
e=\left(c_{\mathrm{e} 1} T+c_{\mathrm{e} 2}\right) \omega \frac{\Delta s}{v}
$$

The electrical power of the traction motor is modeled as a straight line with respect to $T$ and the coefficients $c_{\mathrm{e} 1}$ and $c_{\mathrm{e} 2}$, multiplying the angular velocity $\omega$ of the traction motor as written in Eq. 5.

Under CD mode the cost function only relates to the variables in the first equation in Eq. 3. For CD mode the MPSHC is implemented as a single-input and single-output (SISO) optimization.

The optimal cost function in the case of CS mode is shown below,

$$
J=\sum_{k=1}^{n}\left(f(k)^{2}+\beta e(k)^{2}+\rho\left(u_{1}(k)-u_{\mathrm{L}}(k)\right)^{2}\right)
$$

The variable $f$ in the first term expresses the fuel consumption within $\Delta s$, when the range extender drives the traction motor electrically or charges the battery. In this cost function $f$ and $e$ are supposed to minimize at the same time with the weight $\beta$ in this mode.

The rate of $f$ along the OOL is modeled as a straight line with coefficients $c_{\mathrm{f} 1}$ and $c_{\mathrm{f} 2}$. The fuel consumption $f$ is then expressed as follows,

$$
f=\left(c_{\mathrm{f} 1}\left(e \frac{v}{\Delta s}-p_{\mathrm{b}}\right)+c_{\mathrm{f} 2}\right) \frac{\Delta s}{v}
$$

Then both forms of the optimal cost function are convex. The transition from the $\mathrm{CD}$ to $\mathrm{CS}$ mode can adopt the cost function of the CS mode.

\subsection{Optimization Constraints}

The driving torque is limited by the characteristic of the traction motor, while the braking torque is limited by the maximal allowed deceleration as,

$$
T_{\min }(k) \leq T(k) \leq T_{\max }(k) .
$$


The battery power has its limits as,

$$
P_{\min } \leq p_{\mathrm{b}}(k) \leq P_{\max } .
$$

Here all the optimization constraints for the driving behavior are borrowed from [2]. For the constraint of the speed, namely $x_{1}$ in Eq. 3 , with the speed control, i.e. the roadway ahead is free of traffic, the speed envelope is evaluated by the set speed $v_{\text {set }}$ and the driver given parameter $c_{v s e t}$ as follows,

$$
v_{\min , \max }=v_{\text {set }}\left(1 \mp c_{v s e t}\right) .
$$

When the legal speed limit is met, the speed bounds are overridden. Furthermore the gradient of the speed envelope is limited by a space discrete rate limiter [2]. Therefore the constraint for $x_{1}$ in a horizon is expressed as,

$$
\mathbf{X}_{1 \text { min,max }}=\left(\begin{array}{c}
v_{\text {min,max }}(1)^{2} \\
v_{\text {min,max }}(2)^{2} \\
\vdots \\
v_{\text {min,max }}(n)^{2}
\end{array}\right) .
$$

From Eq. 12 a speed reference $\mathbf{X}_{\mathrm{p}}$ is determined by $\mathbf{X}_{1 \min }, \mathbf{X}_{1 \max }$ and the tunable weight $v$ as follows,

$$
\mathbf{X}_{\mathrm{p}}=\mathbf{X}_{1 \text { max }}-v\left(\mathbf{X}_{1 \text { max }}-\mathbf{X}_{1 \text { min }}\right) .
$$

When $v_{\text {set }}$ is higher than the current speed limit, $v$ is set to zero, and the speed reference is towards the maximal allowed speed. Furthermore, with $\mathbf{X}_{\mathrm{p}}$, the constraint producing the journey time close to the desired value is expressed as,

$$
x_{\text {sum }} \geq \sum_{k=1}^{n} \mathbf{X}_{\mathrm{p}} \text {. }
$$

The variable $x_{\text {sum }}$ in Eq. 13 is the sum of $x_{1}$ in every sampling interval in a horizon. The square root $v_{\mathrm{p}}$ is utilized to replace the speed appearing in the denominators in the model and the cost function, in order to formulate the problem convex,

$$
v_{\mathrm{p}}=\sqrt{\mathbf{X}_{\mathrm{p}}} \text {. }
$$

When there is a preceding object vehicle detected by the radar, the headway control is activated. Here the object vehicle is assumed travelling with constant speed $v_{\mathrm{Obj}}$. The speed envelope is evaluated recursively by the current speed of the subject vehicle $v_{\text {pred }}(1)$ and the current distance $d_{\text {pred }}(1)$ between the subject vehicle and object vehicle as,

$$
\begin{aligned}
& \Delta v_{\text {pred }}(k)=v_{\text {pred }}(k-1)-v_{\text {Obj }} \\
& \Delta t_{\text {pred }}(k)=\frac{\Delta s}{\Delta v_{\text {pred }}(k-1)}, \\
& d_{\text {pred }}(k)=d_{\text {pred }}(k-1)-\Delta v_{\text {pred }}(k-1) \Delta t_{\text {pred }}(k-
\end{aligned}
$$$$
\text { 1), }
$$

$$
v_{\text {pred }}(k)=v_{\text {Obj }}+\delta\left(d_{\text {pred }}(k)-d_{\text {set }}\right) .
$$

The terminal constraint in order to maintain more battery charge/discharge cycles is set to the second state $\mathrm{SoC}$ in the model in Eq. 3 :

$\operatorname{soc}(N+1) \geq S O C_{\min }$.

\section{Simulation Results}

The proposed MPSHC is then integrated in the simulation environment with the double-track vehicle model. The results of the proposed algorithm for different scenarios are explained as follows.

\subsection{The speed control scenario}

As shown in Fig. 2 (a) this scenario has varied speed limits, such as $200 \mathrm{~km} / \mathrm{h}$ and $100 \mathrm{~km} / \mathrm{h}$, as well as the set speed $130 \mathrm{~km} / \mathrm{h}$. The predictive horizon has 20 sampled road segments with $\Delta s=25 \mathrm{~m}$. In the CD mode of the ACC vehicle the electrical energy in the full charged battery is assumed to be used first, until the SoC lower limit is reached. The range extender directly supplies the demanded electrical power for the traction motor in the CS mode.

The ACC controlled speed profile is constant until the lower maximal speed limit is almost reached, and then the vehicle makes a strong brake. The MPSHC controlled vehicle decelerates earlier and with smaller brake torque. When the speed limit is met, with the weight $v$, the vehicle is controlled towards the maximal allowed speed. Overall the electrical energy is saved by $10.8 \%$ with $2.2 \%$ additional journey time under CD mode and the fuel is saved by $10.2 \%$ with $1.8 \%$ additional journey time under the CS mode. Here, as well as later in all the CS modes of the MPSHC in this paper, the electrical energy consumption under CS mode is converted to fuel consumption. For the conversion, the average efficiencies of the range extender module of the CS mode of the ACC in the corresponding scenarios, are put into service. The final $\mathrm{SoC}$ of the CS mode deviates from the terminal constraint $(0.35)$ a little, since the prediction error. A similar situation can be found in the headway control scenario.

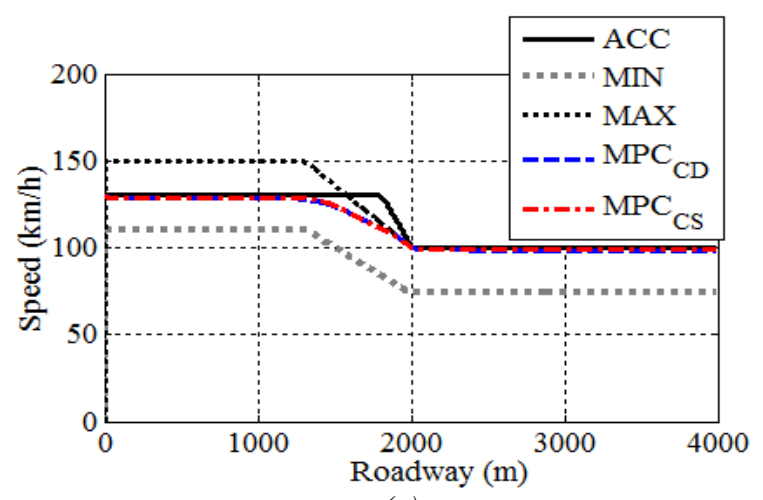

(a) 


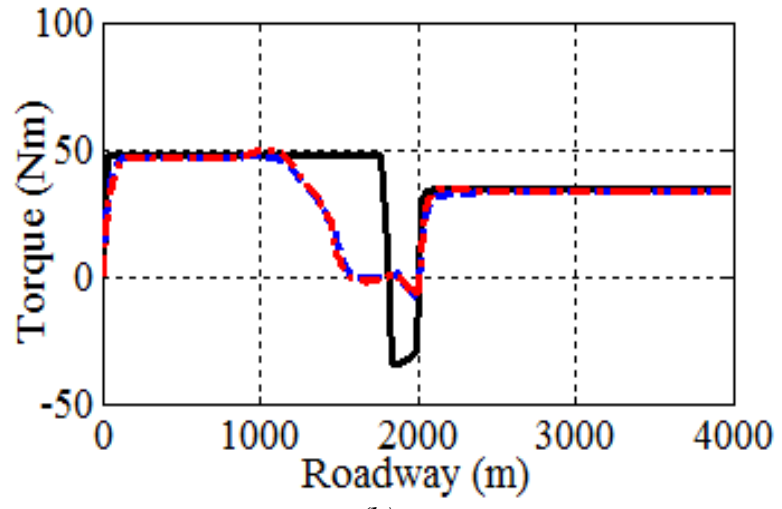

(b)

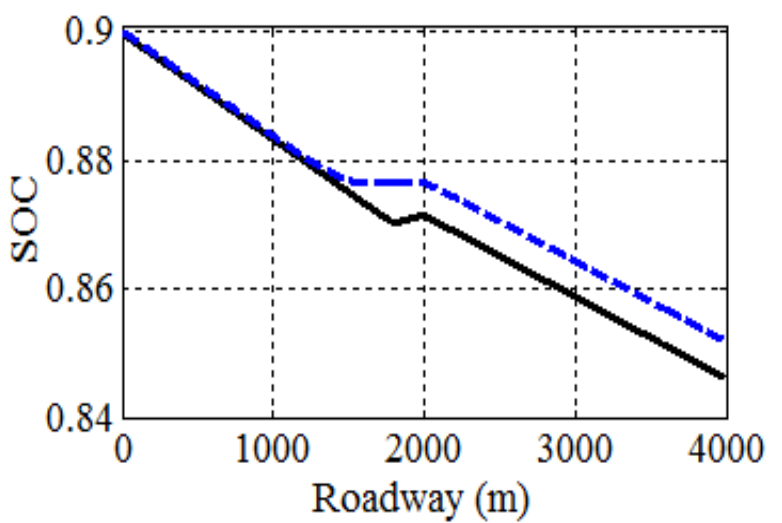

(c)

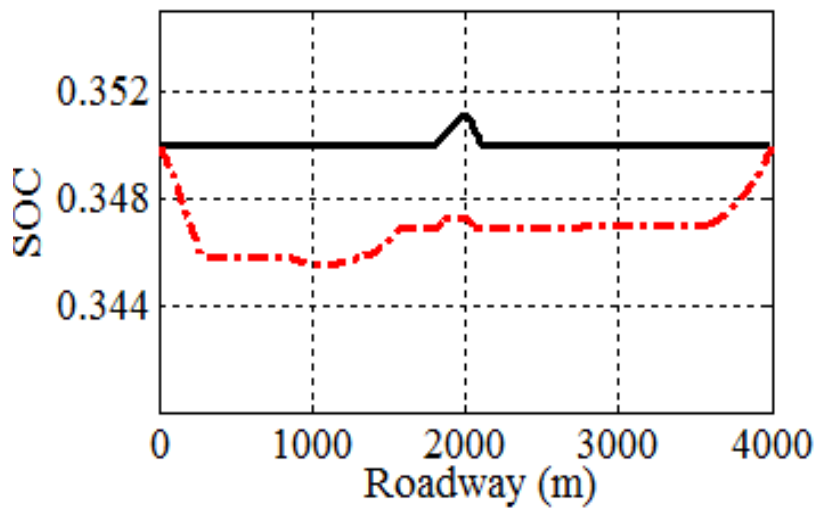

(d)

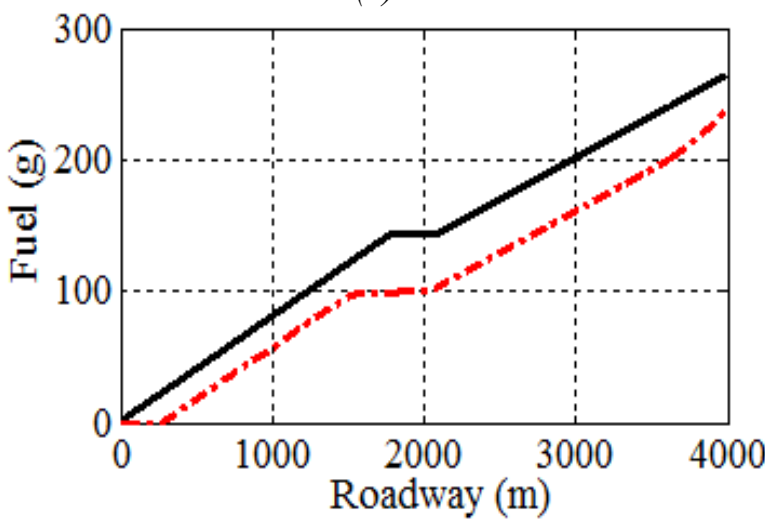

(e)

Figure 2. Simulation results for the speed control scenario: (a) Speed, (b) Motor torque, (c) SoC in CD mode, (d) SoC in CS mode, (e) Fuel consumption in CS mode
In the headway control scenario as shown in Fig. 3 (a), the subject vehicle has a set speed of $150 \mathrm{~km} / \mathrm{h}$. At the beginning the slow preceding object vehicle with an oscillating travelling speed around $120 \mathrm{~km} / \mathrm{h}$ comes into the radar range $(200 \mathrm{~m})$. The limits for the MPSHC time gap are $1.5 \mathrm{~s} . .3 .5 \mathrm{~s}$, or rather $48 \mathrm{~m} . .122 \mathrm{~m}$ between subject and object vehicles. The speed of the preceding object vehicle is depicted in light grey in Fig. 3 (a). Furthermore in Fig. 3 (a) the MPSHC controlled vehicle starts to decelerate much earlier. It is obvious, that the MPSHC controlled vehicle decelerates with much smaller brake at the beginning as seen in Fig. 3 (b). The driving torque is with smaller oscillation compared to the ACC controlled vehicle. In this scenario, the MPSHC saves 5.2\% of electrical energy with an additional journey time of $0.04 \%$ under CD mode and $5.9 \%$ of fuel under the CS mode without additional journey time.

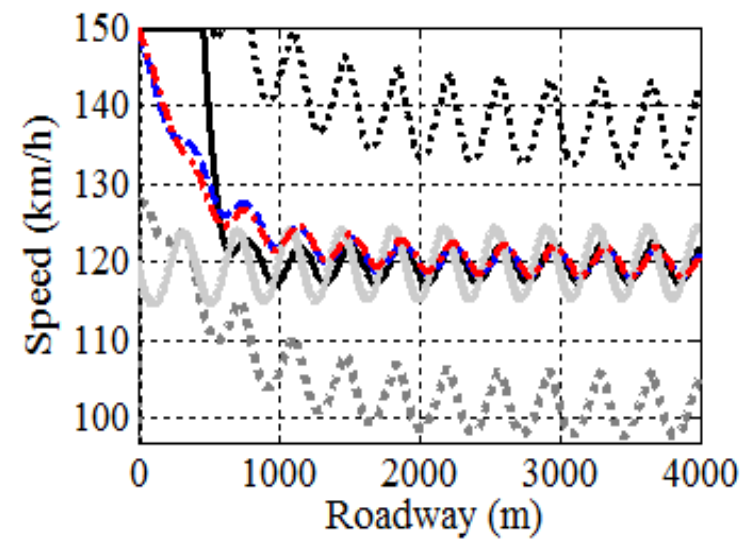

(a)

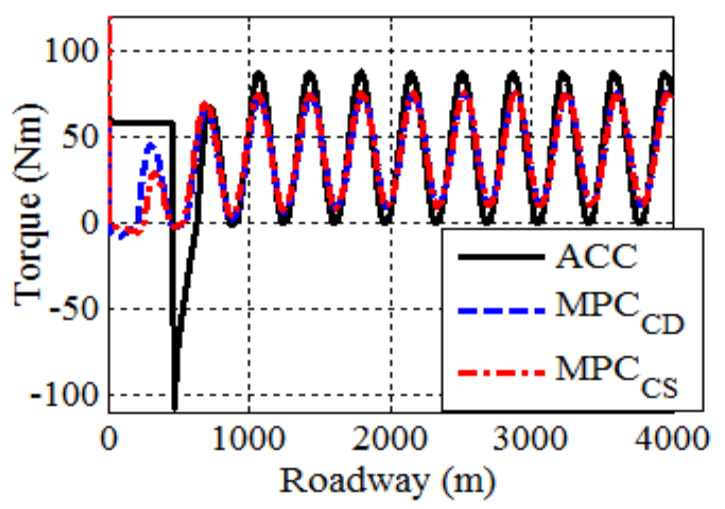

(b)

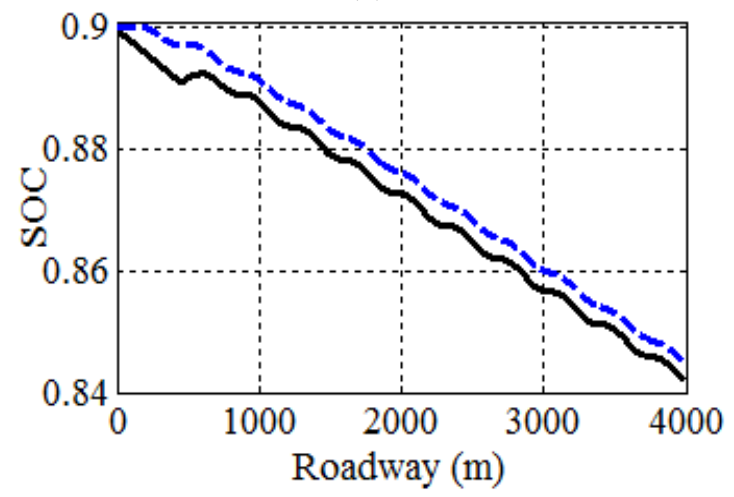

(c)

\subsection{Headway Control Scenario}




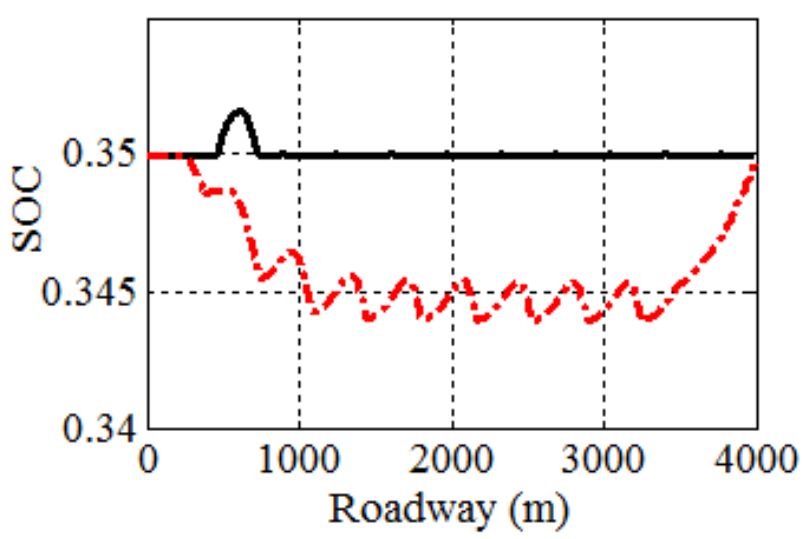

(d)

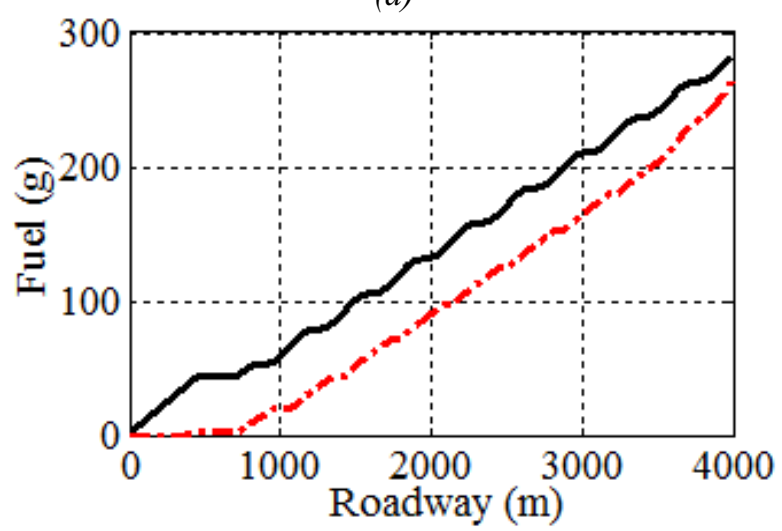

(e)

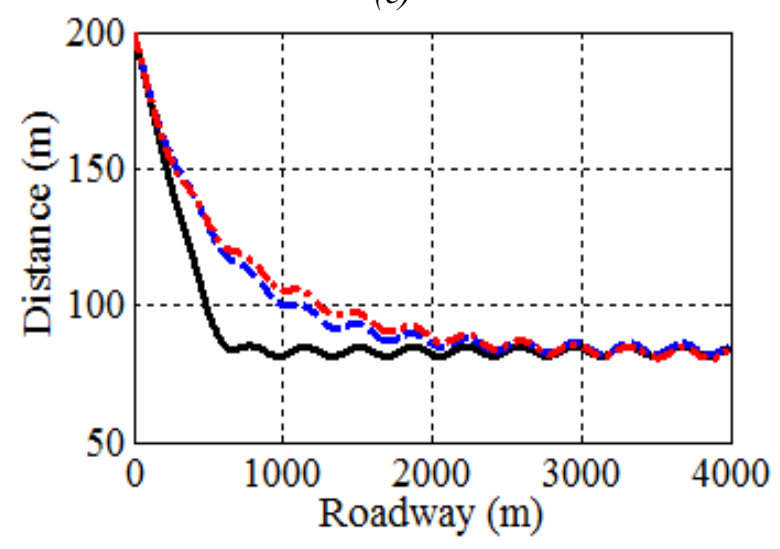

(f)

\section{Summary}

In the speed and headway control scenarios with MPSHC, either the electrical energy or the fuel is saved with a moderate increase of the journey time and acceptable deviations from the set speeds and set distances to the preceding object vehicle. The first numerical simulation results show the reasonable optimized speed and torque trajectories.

In the future work the more sophisticated method for fuel consumption correction in the CS mode of the MPSHC should be adopted.

\section{References}

1. M. Kalabis and S. Müller: A Model Predictive Control Algorithm to Improve Fuel Efficiency of Adaptive Cruise Control Systems, in Proc. 10th International Symposium on Advanced Vehicle Control (AVEC2010), Loughborough, (2010).

2. M. Kalabis and S. Müller: A Model Predictive Headway Controller to Increase Fuel Efficiency Including Gearshift and Braking Strategies, in Proc. 22nd IAVSD Symposium, Manchester, (2011), p. 14-19.

3. T. Schwickart and H. Voos, Driver Assistance System for Predictive Energy Efficient Speed Control Especially for Electric Vehicles (Fahrerassistenzsystem zur vorausschauenden energieeffizienten Geschwindigkeitsregelung speziell für Elektrofahrzeuge), in Proc. VDI Conference on Driver Assistance Systems, Wolfsburg, (2014), p. 57-64.

4. T. Chen, Y. Luo and K. Li, Multi-Objective Adaptive Cruise Control Based on Nonlinear Model Predictive Algorithm, in Proc. IEEE the International Conference on Vehicular Electronics and Safety (ICVES), Peking, (2011).

5. J. Löfberg. YALMIP: A Toolbox for Modeling and Optimization in MATLAB, in Proc. the CACSD Conference, Taipei, Taiwan, (2004).

6. https://www.mosek.com

Figure 3. Simulation results for the headway control scenario: (a) Speed, (b) Motor torque, (c) SoC in CD mode, (d) SoC in CS mode, (e) Fuel consumption in CS mode, (f) Distance 